\title{
Preliminary interpretation of Titan plasma interaction as observed by the Cassini Plasma Spectrometer: Comparisons with Voyager 1
}

\author{
R. E. Hartle, ${ }^{1}$ E. C. Sittler Jr., ${ }^{1}$ F. M. Neubauer, ${ }^{2}$ R. E. Johnson, ${ }^{3}$ H. T. Smith, ${ }^{3}$ F. Crary, ${ }^{4}$ \\ D. J. McComas, ${ }^{4}$ D. T. Young, ${ }^{4}$ A. J. Coates, ${ }^{5}$ D. Simpson, ${ }^{1}$ S. Bolton, ${ }^{6}$ D. Reisenfeld, ${ }^{7}$ \\ K. Szego, ${ }^{8}$ J. J. Berthelier, ${ }^{9}$ A. Rymer, ${ }^{5}$ J. Vilppola, ${ }^{10}$ J. T. Steinberg, ${ }^{11}$ and N. Andre ${ }^{12}$ \\ Received 29 September 2005; revised 29 November 2005; accepted 12 January 2006; published 20 April 2006.
}

[1] The Cassini Plasma Spectrometer (CAPS) instrument observed the plasma environment at Titan during the Cassini orbiter's TA encounter on October 26, 2004. Titan was in Saturn's magnetosphere during the Voyager 1 flyby and also during the TA encounter. CAPS measurements from this encounter are compared with measurements made by the Voyager 1 Plasma Science Instrument (PLS). The comparisons focus on the composition and nature of ambient and pickup ions. They lead to: A) the major ion components of Saturn's magnetosphere in the vicinity of Titan are $\mathrm{H}^{+}, \mathrm{H}_{2}^{+}$and $\mathrm{O}^{+} / \mathrm{CH}_{4}^{+}$ions; B) finite gyroradius effects are apparent in ambient $\mathrm{O}^{+}$ions as the result of their absorption by Titan's extended atmosphere; C) the principal pickup ions are composed of $\mathrm{H}^{+}, \mathrm{H}_{2}^{+}, \mathrm{N}^{+} / \mathrm{CH}_{2}^{+}, \mathrm{CH}_{4}^{+}$, and $\mathrm{N}_{2}^{+}$; D) the pickup ions are in narrow energy ranges; and $\mathrm{E}$ ) there is clear evidence of the slowing down of background ions due to pickup ion mass loading. Citation: Hartle, R. E., et al. (2006), Preliminary interpretation of Titan plasma interaction as observed by the Cassini Plasma Spectrometer: Comparisons with Voyager 1, Geophys. Res. Lett., 33, L08201, doi:10.1029/2005GL024817.

\section{Introduction}

[2] Voyager 1 plasma and field instruments detected a complex interaction between Titan's atmosphere and Saturn's outer magnetosphere [Bridge et al., 1981; Ness et al., 1981]. These initial results were followed by more comprehensive analysis [Hartle et al., 1982, hereinafter referred to as H82; Neubauer et al., 1984]. A new picture of the interaction of Saturn's sub-rotating magnetospheric plasma with Titan's atmosphere emerged from the field and particle measurements as Voyager 1 flew by Titan on November 12, 1980. Since then a number of atmosphere, ionosphere and interaction models [e.g., see Keller et al., 1998] have been developed that encouraged further analysis of the data. This stimulated Sittler et al. [2005] (hereinafter referred to as $\mathrm{S} 05)$ to revisit the Voyager 1 results. Their analysis concluded that pickup ions $\mathrm{H}^{+}$and $\mathrm{H}_{2}^{+}$dominate at

\footnotetext{
${ }^{1}$ NASA Goddard Space Flight Center, Greenbelt, Maryland, USA.

${ }^{2}$ Institute for Geophysics and Meteorology, University of Cologne, Cologne, Germany.

${ }^{3}$ Department of Engineering, University of Virginia, Charlottesville, Virginia, USA.

${ }^{4}$ Southwest Research Institute, San Antonio, Texas, USA.

${ }^{5}$ Mullard Space Flight Center, Dorking, UK.

${ }^{6}$ Jet Propulsion Laboratory, Pasadena, California, USA.

Copyright 2006 by the American Geophysical Union. 0094-8276/06/2005GL024817
}

the largest distances from Titan, followed by $\mathrm{CH}_{4}^{+}$at intermediate distances and $\mathrm{N}_{2}^{+}$just outside the magnetopause [Neubauer et al., 1984]. The relative abundance of these ions is consistent with the densities of the corresponding neutral exosphere constituents. Finite gyroradius effects were found to be important to the plasma interaction with Titan's atmosphere, resulting in removal of ambient ions by Titan's upper atmosphere. The analysis implied that the observed hot $\mathrm{keV}$ ambient ion component, $\mathrm{N}^{+} / \mathrm{O}^{+}$, was removed.

[3] In Figure 1, the Cassini flyby trajectory in Titancentered coordinates is super-imposed on the model interaction originally derived by $\mathrm{H} 82$ from Voyager 1 data. This 'view' in the $-\mathrm{z}$ direction shows that the Voyager 1 trajectory is similar to that of the TA encounter, except that Voyager 1 is further down the wake region. For TA, the closest approach altitude was $\mathrm{z} \sim 1174 \mathrm{~km}$ from the surface at 15:30:08 SCET (spacecraft event time in [hr:min:s] throughout). The TA encounter was $\sim 10.6 \mathrm{hr}$ LT relative to Saturn and Voyager 1 was $\sim 13 \mathrm{hr}$ LT. The similarities of these flyby trajectories allows direct comparison of CAPS and PLS plasma measurements.

[4] The CAPS instrument [Young et al., 2004] is composed of an Ion Mass Spectrometer (IMS), Electron Spectrometer (ELS) and an Ion Beam Spectrometer (IBS). We will primarily be discussing data from the IMS. The IBS is most important for Titan's ionosphere. The IMS covers the ion energy-per-charge range $1 \mathrm{~V} \leq \mathrm{E} /$ $\mathrm{Q} \leq 50 \mathrm{kV}$. The IMS (and ELS) takes simultaneous measurements in collimators divided into 8 angular sectors separated by $20^{\circ}$ in the collimator plane. The IMS measures E/Q spectra, called singles, at non-coincident ion rates (no composition information). Simultaneously, the IMS takes coincident time-of-flight (TOF) data (or B-cycle data, E/Q vs TOF) used for detailed compositional analysis. An IMS energy sweep takes 4 seconds and the B-cycle takes 256 seconds. For the B-cycle data we sum 64 energy sweeps and collapse all 8 angular sectors for high sensitivity. An actuator sweeps the field of view of the sensors to optimize pitch angle coverage. The actuator was scanned at a rate of $1^{\circ} \mathrm{s}^{-1}$ over a

\footnotetext{
${ }^{7}$ Department of Physics and Astronomy, University of Montana, Missoula, Montana, USA.

${ }^{8}$ KFKI Research Institute for Particle and Nuclear Physics, KFKIRMKI, Budapest, Hungary.

${ }^{9}$ Centre d'etude des Environnements Terrestre et Planetaires, St. Maurdes-Fosses, France.

${ }^{10}$ Department of Physical Sciences, University of Oulu, Linnanmaa, Finland.

${ }^{11}$ Los Alamos National Laboratory, Los Alamos, New Mexico, USA.
${ }^{12}$ Centre d'Etude Spatiale des Rayonnements, Toulouse, France.
} 


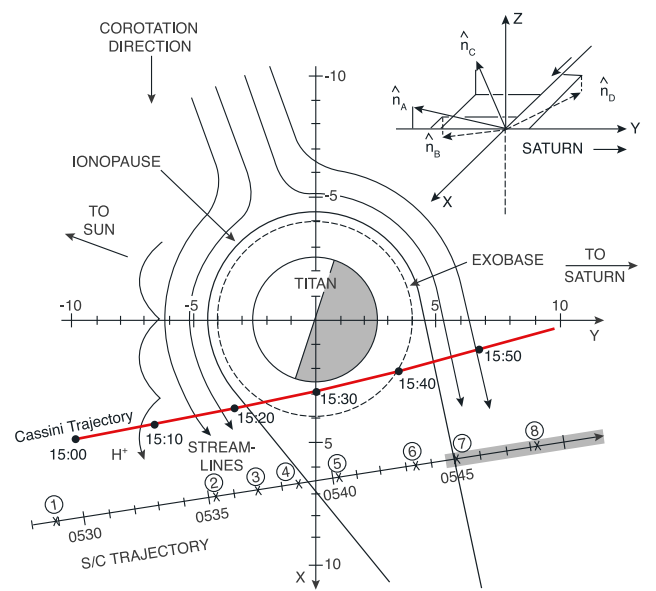

Figure 1. Geometrical properties of Titan interaction projected onto orbital plane $(\mathrm{x}, \mathrm{y})$. Interval spacing on $\mathrm{x}$ and $\mathrm{y}$-axes are in intervals of $10^{3} \mathrm{~km}$. On the Voyager 1 trajectory, the numbers are positions where PLS ion measurements were made, while the IMS measurements were made almost continuously along the Cassini trajectory. The Voyager 1 trajectory is inclined $\sim 10^{\circ}$ to the $\mathrm{x}-\mathrm{y}$ plane, while TA is inclined $\sim 17.3^{\circ}$. Voyager 1 crosses the $\mathrm{x}-\mathrm{y}$ plane from above at $0542 \mathrm{UT}$. TA does not cross the $x-y$ plane in the magnetotail; however, for orientation, it crossed the terminator plane above the equatorial plane at 15:29:59 UT. (dashed line) Voyager 1 model exobase and (solid line) ionopause boundaries from H82 are shown along with streamline flow and an $\mathrm{H}^{+}$ pickup ion trajectory (to scale). The inset in the upper right shows the directions of the normal vectors to the 4 PLS Faraday cups.

range of $\sim+107^{\circ}$ to $\sim-81^{\circ}$ during Titan-A, with a sweep across the ram direction for the immediate Titan encounter.

\section{Flow and Composition}

[5] Figure 2 shows the E/Q vs. time spectrogram of IMS singles for all 8 angular sectors during the TA encounter, which is used to give an overview of plasma flow and composition. The background plasma outside Titan's interaction region has been analyzed by F. Crary et al. (Dynamics and composition of plasma at Titan, submitted to Science, 2005, hereinafter referred to as Crary et al., submitted manuscript, 2005). They find, outside the Titan interaction region, ion distributions with two peaks, having $\mathrm{E} / \mathrm{Q} \sim 200 \mathrm{~V}$ and $\mathrm{E} / \mathrm{Q} \sim 2 \mathrm{kV}$, consistent with $\mathrm{H}^{+}-\mathrm{H}_{2}^{+}, \mathrm{N}^{+} / \mathrm{CH}_{2}^{+}$and $\mathrm{O}^{+} / \mathrm{CH}_{4}^{+}$ions flowing at $\sim 110 \pm$ $20 \mathrm{~km} \mathrm{~s}^{-1}$ (corotation speed $\sim 200 \mathrm{~km} / \mathrm{s}$ ), where the ion masses were identified from counts vs TOF data. From $\sim 15: 00$ to $\sim 15: 18 \mathrm{SCET}$, the spacecraft was in the pickup region, where the $\mathrm{E} / \mathrm{Q}$ range decreased to $\sim 5-50 \mathrm{~V}$ and $\mathrm{N}_{2}^{+}$has speeds $\sim 10-15 \mathrm{~km} / \mathrm{s}$, which is an ion suggested by the TOF data below and identified by H82, S05 and Crary et al. (submitted manuscript, 2005). From $\sim 13: 00$ to $\sim 15: 18$ SCET, the greatest ion counts occurred in the three lowest sectors, which are also the ones pointing most closely into the corotation direction of the magnetosphere. The sectors sequentially beyond the third point upstream with increasing components toward Saturn. From 15:22 to 15:40 SCET, the spacecraft was within Titan's ionotail and passed through the ionosphere (described by Crary et al. (submitted manuscript, 2005)). After 15:40 SCET the spacecraft exited the ionosphere and quickly returned to the dynamical conditions of Saturn's magnetosphere plasma. Following a spacecraft roll that ended about 16:00 SCET, the greatest ion counts occurred in the second through fifth sectors from the top, which are also the ones pointing most closely into the corotation direction. In contrast to that on the anti-Saturn side, this rapid change is due to the lack of sufficient pickup ions to mass load the plasma. The paucity of pickup ions on the Saturn facing side occurs because the cycloidal trajectories of the heavy ions intercept Titan's atmosphere, where most of them are absorbed. Well outside the pickup region on the anti-Saturn side, the heavy ion composition favors $\mathrm{O}^{+} /$ $\mathrm{CH}_{4}^{+}$. The plasma interaction region described above essentially coincides with the induced magnetosphere from $\sim 15: 10$ to 15:40 SCET [Backes et al., 2005].

[6] We have made an additional analysis of magnetospheric ion composition using CAPS E/Q vs TOF spectrograms obtained from $12: 30$ to $14: 00$ SCET, in the vicinity of Titan, but well away from the mass loading region. During this long observation interval, counts were integrated over many B cycles. Spectral "lines" for ion masses 1, 2 and 16 amu are identified and interpreted to be $\mathrm{H}^{+}, \mathrm{H}_{2}^{+}$and $\mathrm{O}^{+}$. This ambient plasma is hot, having energy ranges of $\sim 0.016-6.3 \mathrm{keV}$ for $\mathrm{H}^{+}, \sim 0.1-4 \mathrm{keV}$ for $\mathrm{H}_{2}^{+}$and $\sim 1-4 \mathrm{keV}$ for $\mathrm{O}^{+}$. The identification of $\mathrm{H}^{+}$

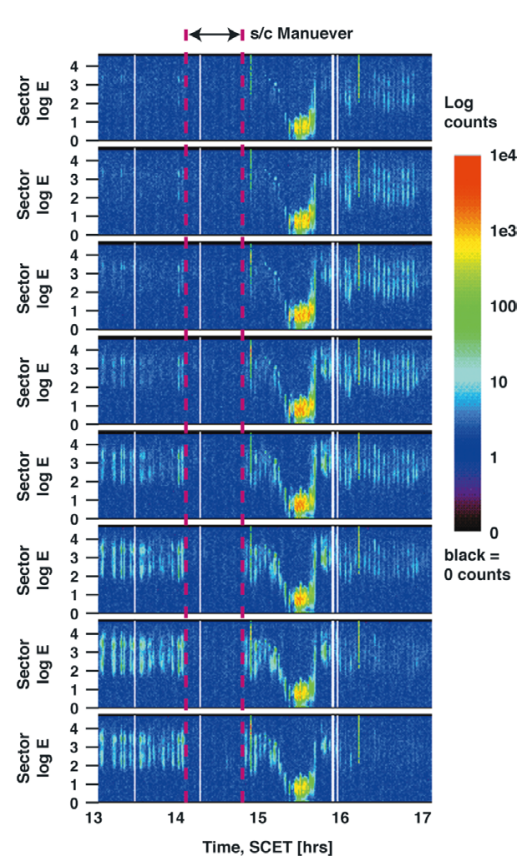

Figure 2. IMS singles (non-coincident ion rates, i.e., no composition information) spectrogram observed on TA trajectory. All 8 angular sectors are shown as energy per charge, E/Q [eV] versus SCET (in [hr:min:s] throughout) versus counts depicted by color scale in logarithmic counts to right. Discrete energy scans appear as vertical swaths. The white vertical lines are data gaps. 


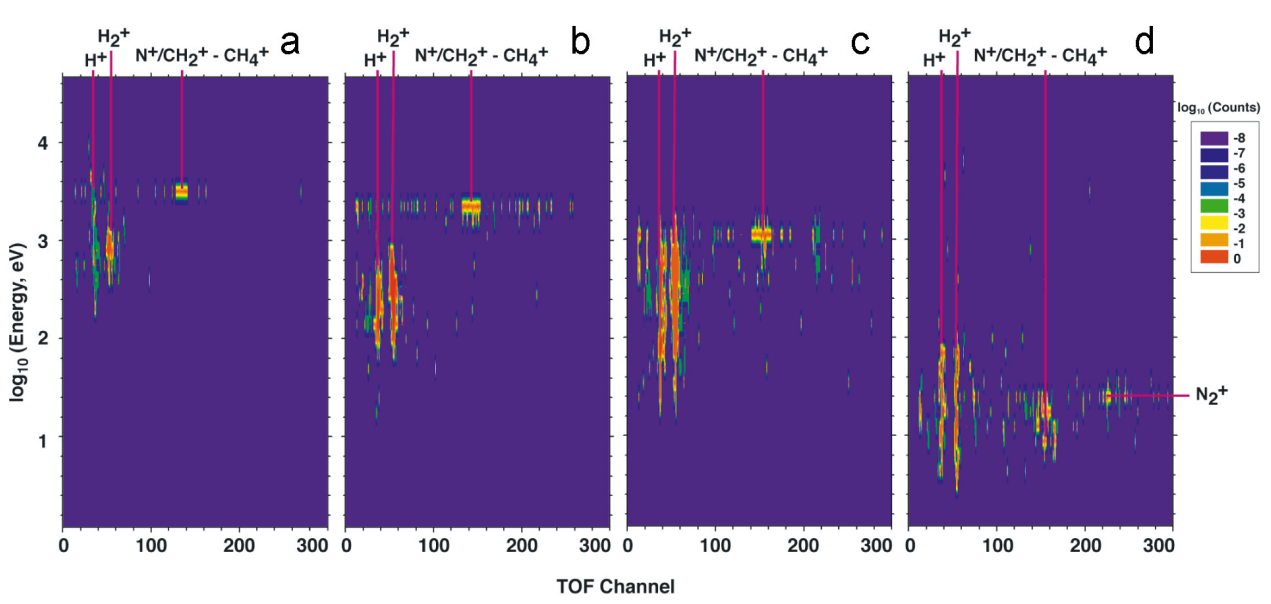

Figure 3. IMS coincident TOF spectrogram observed on TA trajectory and shown as energy per charge, E/Q [eV] versus TOF channels versus counts depicted by color scale in logarithmic counts to right. The spectrograms in $3 \mathrm{a}-3 \mathrm{~d}$ are single B cycles observed sequentially and derived from the mass loading region preceding entry into Titan's ionosphere. The $4 \mathrm{~B}$ cycles (each taking $\sim 4 \mathrm{~min}$ ) start at SCET's: (a) 15:02:00, (b) 15:06:16, (c) 15:10:32 and (d) $15: 14: 48$.

and $\mathrm{O}^{+}$is strengthened by the appearance of $\mathrm{H}^{-}$and $\mathrm{O}^{-}$ lines, their instrumental "fingerprints" [Young et al., 2004].

[7] S05 used a revised model neutral exosphere for source calculations of pickup ions. New species not included $\mathrm{H} 82$ are $\mathrm{H}_{2}, \mathrm{CH}_{4}$, and suprathermal $\mathrm{N}^{*}$. They were added S05, including the corresponding ionization rates, to study pickup ion mass loading. It was also pointed out by S05 that, as Voyager 1 approached Titan, the high energy part of the spectrum disappeared first, identified as the heavy component $\mathrm{N}^{+} / \mathrm{O}^{+}$by $\mathrm{H} 82$, and then the lower energy component, $\mathrm{H}^{+}$, disappeared when the spacecraft was close to the magnetopause of Titan. On exiting the magnetotail the light ion component recovered first followed by the heavy, more energetic component. This was shown to be a finite gyroradius effect, where ambient $\mathrm{H}^{+}$and $\mathrm{N}^{+} / \mathrm{O}^{+}$are absorbed by the atmosphere on terminator streamlines about $800 \mathrm{~km}$ and $11,200 \mathrm{~km}$ above the exobase $(4,000 \mathrm{~km})$, the sizes of their ambient gyrodiameters, respectively. The CAPS data below supports these interpretations.

[8] TOF spectrograms in the mass loading region are shown in Figure 3 for 4 B cycle time intervals $(\sim 4 \mathrm{~min})$ starting at SCET's: 15:02:00, 15:06:16, 15:10:32 and 15:14:48. CAPS mass resolution is $\mathrm{m} / \Delta \mathrm{m} \sim 8$. For reference, the Cassini magnetometer observed the beginning of magnetic field draping at $~ 15: 10$ SCET, occurring just before the third B cycle [Backes et al., 2005]. Spectral signatures of $\mathrm{H}^{+}$and $\mathrm{H}_{2}^{+}$along with the accompanying $\mathrm{H}^{-}$ are identified in Figure 3. The ion energy ranges in the first B cycle, Figure $3 \mathrm{a}$, are $\sim 0.13-3.4 \mathrm{keV}$ for $\mathrm{H}^{+}$and $\sim 0.3-$ $1.2 \mathrm{keV}$ for $\mathrm{H}_{2}^{+}$. Considering the presence of a neutral exospheric source for these ions, their spectra are a mixture of ambient and pickup ions. In contrast, the heavier ion spectra in the range of $14-16$ amu are observed in a narrower energy range of $\sim 3.4-4.0 \mathrm{keV}$. Of particular interest is the missing ambient $\mathrm{O}^{+}$fingerprint, $\mathrm{O}^{-}$, which is consistent with the absorption of $\mathrm{O}^{+}$by Titan's atmosphere on those magnetosphere plasma flux tubes inside about a gyrodiameter of the Titan's exobase. It follows that the atmosphere should also absorb any ambient $\mathrm{N}^{+} / \mathrm{CH}_{2}^{+}$, and $\mathrm{CH}_{4}^{+}$present. We identify the remaining $14-16 \mathrm{amu}$ ions as pickup ions, where the $16 \mathrm{amu}$ ion is identified as $\mathrm{CH}_{4}^{+}$since methane is the dominant 16 amu exospheric constituent ( $\mathrm{S} 05)$ as observed by the Ion Neutral Mass Spectrometer on Cassini [Waite et al., 2005]. The mass 14 amu pickup ion could be a mixture of $\mathrm{N}^{+}$and $\mathrm{CH}_{2}^{+}$, where the former is the dominant 14 amu exospheric gas and the latter ion is a fragment of its parents' $\mathrm{CH}_{4}$ and $\mathrm{CH}_{4}^{+} . \mathrm{N}_{2}^{+}$is identified as the $28 \mathrm{amu}$ ion in the fourth B-cycle. Possible ions of ionospheric origin [Keller et al., 1998] are $\mathrm{CH}_{3}^{+}$(between the 14-16 amu channels), $\mathrm{HCNH}^{+}$, and $\mathrm{CH}_{5}^{+}$and $\mathrm{C}_{2} \mathrm{H}_{5}^{+}$(weak indications at TOF channels above the 16 and 28 amu channels). These ionospheric ions may be scavenged by the interaction of the externally flowing plasma with the ionosphere, a well known process observed at Venus.

[9] Atmospheric capture of background plasma was discussed by $\mathrm{H} 82$ and S05. CAPS measurements show that magnetospheric $\mathrm{O}^{+}$is not present inside $\sim 12,500 \mathrm{~km}$ from the $\mathrm{x}$-axis, a region where $\mathrm{O}^{-}$is absent from the $\mathrm{B}$ cycle starting at 14: 53 SCET and continuing at all points inward. This is outside the region where any noticeable magnetospheric deflection of flow around Titan has been observed (e.g., slowing down begins after Figure $3 \mathrm{a}$ as does the first indication of magnetic field line draping [Backes et al., 2005]). Taking this as the edge of the $\mathrm{O}^{+}$clearing area, the ion gyrodiameter between this point and the exobase $(4,000 \mathrm{~km})$ is $8,500 \mathrm{~km}$. The corresponding guiding center is $8,250 \mathrm{~km}$ from the $\mathrm{x}$-axis. For an initial estimate, we define a circular $\mathrm{O}^{+}$clearing area of radius $8,250 \mathrm{~km}$, which intercepts all guiding centers out to the empirically derived outer edge. The 16 amu background ions were observed to be in a shell distribution by Szego et al. [2005] and its interaction characteristics were modeled by Ledvina et al. [2005]. As a magnetic flux tube of the same area flows past Titan at $110 \mathrm{~km} \mathrm{~s}^{-1}$, its time-averaged area intercepting the clearing area is $1.4 \times 10^{18} \mathrm{~cm}^{2}$. Then, using the clearing 
area, the $\mathrm{O}^{+}$density, $0.06 \mathrm{~cm}^{-3}$ (Crary et al., submitted manuscript, 2005), the background flow speed, $110 \mathrm{~km} \mathrm{~s}^{-1}$ and a thermal speed, $110 \mathrm{~km} \mathrm{~s}^{-1}$ (conservative estimate since flow is subsonic), we obtain an $\mathrm{O}^{+}$loss rate to Titan of $\sim 9 \times 10^{23} \mathrm{~s}^{-1}$.

\section{Mass Loading}

[10] As Cassini moved closer to Titan and further into the mass loading region, a moderate decrease in ion energies occurred as shown in Figures $3 a-3 c$. Average energy decreases of about 1.5 and 2 times occurred in the 14-16 amu group, respectively. A much larger energy drop of $\sim 125$ times occurred between the 3rd and 4th B cycles of Figures $3 \mathrm{c}$ and $3 \mathrm{~d}$, respectively. This large energy decrease is attributed to the increase in the number of pickup ions added to the flow (as the neutral exosphere source densities increase) and the addition of the heavier $28 \mathrm{amu}$ ion, initially identified as $\mathrm{N}_{2}^{+}$, reflecting the increasing density of the exospheric source upstream. The ion energies for the $28 \mathrm{amu}$ ion in the 4th B cycle correspond to flow speeds $\sim 10-15 \mathrm{~km} \mathrm{~s}^{-1}$. This highly decelerated plasma is the last cycle before the cooler, denser ionospheric plasma is observed [Coates et al., 2005].

[11] Effects of mass loading on ambient flow speeds in the pickup region were computed by $\mathrm{S} 05$. The analysis was guided by comparisons with Voyager 1 plasma ion measurements. The CAPS observations above are supportive of the mass loading picture observed by PLS on Voyager 1 (H82) and the reduced flow speeds.

\section{Ion Energy Distributions}

[12] As described by H82, the newly born pickup ions are accelerated by the motional electric field, $\mathbf{E}=-\mathbf{V} \times$ B, pointing away from Saturn when in Saturn's equatorial plane, where $\mathbf{V}$ is the background velocity of Saturn's rotating magnetosphere and $\mathbf{B}$ is its magnetic field. The ions move in a plane perpendicular to $\mathbf{B}$, which is also perpendicular to the plane containing both $\mathbf{V}$ and $\mathbf{B}$. At distances well away from Titan, newly born pickup ions move in cycloidal trajectories in planes approximately parallel to Saturn's equatorial plane and their initial distribution in velocity space is a ring distribution. Because the ion gyroradii of the 14-16 amu ions are much larger than their neutral source gas scale heights, $\mathrm{H}$, the majority of the ions observed were born over small spatial regions relative their gyroradii, initially confining them to only a fraction of the allowable velocity space [Hartle and Sittler, 2004]. In this case, such heavy ions are expected to appear as narrow beams in configuration, velocity and energy space. The spectra of the 14-16 amu ions in Figures $3 \mathrm{a}$ and $3 \mathrm{~b}$ appear to be consistent with the signatures of such pickup ion beams. As the magnetic field begins to drape around Titan, the beam trajectories leave the equatorial plane. The field was observed to start draping at $\sim 15: 10$ SCET, just before the B cycle of $3 \mathrm{c}$ [Backes et al., 2005]. The draping becomes more prominent during the $\mathrm{B}$ cycle of $3 \mathrm{~d}$; however, the energy spread remained similar to the previous panels, suggesting that the beam remained coherent. We use the actuator motion of the IMS in the mass loading region to aid in studying pickup ion distributions. As the actuator continuously sweeps above and below the equatorial plane, the collimator should accept ion beams over a segment of its angular sweep. If the ions were not confined as a beam, they would be observed over wider angular segments. Furthermore, the pickup ion energies "jump" to lower energy ranges on passing from Figures $3 \mathrm{a}$ to $3 \mathrm{~d}$ or $4 \mathrm{~B}$ cycles. Such jumps are expected for a narrow beam distribution because the collimator makes only one pass through the equatorial plane during each $\mathrm{B}$ cycle, assuring the likelihood that an ion beam would be crossed. The energy jump occurs because the plasma speed continuously decreased during $\sim 4$ min of each $\mathrm{B}$ cycle while the distribution was only observed during that portion of the cycle when the collimator accepted an ion beam. Other distributions would have energies outside that of a beam. Shell and Maxwellian distributions moving at subsonic speeds would appear with broader energy ranges, including many at lower energies.

\section{Summary}

[13] The general features of the interaction of Saturn's rotating magnetosphere with Titan's atmosphere observed by CAPS during the Cassini TA flyby are similar to those observed by PLS on Voyager 1. A mass loading region is observed during the inbound legs of these flybys, where pickup ions are observed in narrow energy ranges consistent with ion beams. In this region, the plasma is slowed down from $\sim 110 \mathrm{~km} \mathrm{~s}^{-1}$ to $\sim 10-15 \mathrm{~km} \mathrm{~s}^{-1}$. Saturn's ambient ions, dominated by $\mathrm{O}^{+}$and $\mathrm{CH}_{4}^{+}$, are removed in this region by the atmosphere. The pickup ions are composed of $\mathrm{H}^{+}$, $\mathrm{H}_{2}^{+}, \mathrm{N}^{+} / \mathrm{CH}_{2}^{+}, \mathrm{CH}_{4}^{+}$, and $\mathrm{N}_{2}^{+}$, where the latter two do most of the mass loading.

\section{References}

Backes, H. F., et al. (2005), Titan's magnetic field signature during the first Cassini encounter, Science, 308, 992-995.

Bridge, H. S., et al. (1981), Plasma observations near Saturn: Initial results from Voyager 1, Science, 212, 217-224.

Coates, A. J., et al. (2005), Cassini's first Titan encounters: A comparison of plasma results, Geophys. Res. Abstr., 7, Abstract 08841.

Hartle, R. E., and E. C. Sittler Jr. (2004), Pickup ion velocity distributions at Titan: Effects of spatial gradients, Eos Trans. American Geophysical Union, 85(17), Jt. Assem. Suppl., Abstract P33D-04.

Hartle, R. E., E. C. Sittler Jr., K. W. Ogilvie, J. D. Scudder, A. J. Lazarus, and S. K. Atreya (1982), Titan's ion exosphere observed from Voyager 1, J. Geophys. Res., 87, 1383-1394.

Keller, C. N., V. G. Anicich, and T. E. Cravens (1998), Model of Titan's ionosphere with detailed hydrocarbon ion chemistry, Planet. Space Sci., 46, $1157-1174$.

Ledvina, S. A., T. E. Cravens, and K. Kecskeméty (2005), Ion distributions in Saturn's magnetosphere near Titan, J. Geophys. Res., 110, A06211, doi:10.1029/2004JA010771.

Ness, N. F., M. H. Acuna, R. P. Lepping, J. E. P. Connerney, K. W. Behannon, L. F. Burlaga, and F. M. Neubauer (1981), Magnetic field studies by Voyager 1: Preliminary results at Saturn, Science, 212, $211-$ 217.

Neubauer, F. M., D. A. Gurnett, J. D. Scudder, and R. E. Hartle (1984), Titan's magnetospheric interaction, in Saturn, edited by T. Gehrels and M. S. Matthews, pp. 760-787, Univ. of Ariz. Press, Tucson.

Sittler, E. C., Jr., R. E. Hartle, A. F. Viñas, R. E. Johnson, H. T. Smith, and I. Mueller-Wodarg (2005), Titan interaction with Saturn's magnetosphere: Voyager 1 results revisited, J. Geophys. Res., 110, A09302, doi:10.1029/ 2004JA010759.

Szego, K., et al. (2005), The global plasma environment of Titan as observed by Cassini Plasma Spectrometer during the first two close encounters with Titan, Geophys. Res. Lett., 32, L20S05, doi:10.1029/ 2005 GL022646. 
Waite, J. H., Jr., et al. (2005), Ion neutral mass spectrometer results from the first flyby of Titan, Science, 308, 982-986.

Young, D. T., et al. (2004), Cassini plasma spectrometer investigations, Space Sci. Rev., 114, 1-112.

N. Andre, Centre d'Etude Spatiale des Rayonnements, F-31028 Toulouse, France.

J. J. Berthelier, Centre d'etude des Environnements Terrestre et Planetaires, F-94107 St. Maur-des-Fosses, France.

S. Bolton, Jet Propulsion Laboratory, Pasadena, CA 91109, USA.

A. J. Coates and A. Rymer, Mullard Space Flight Center, Dorking RH5 6NT, UK.

F. Crary, D. J. McComas, and D. T. Young, Southwest Research Institute, San Antonio, TX 78228-0510, USA.
R. E. Hartle, D. Simpson, and E. C. Sittler, NASA Goddard Space Flight Center, Greenbelt, MD 20771, USA. (richard.e.hartle@nasa.gov)

R. E. Johnson and H. T. Smith, Department of Engineering, University of Virginia, Charlottesville, VA 22904, USA.

F. M. Neubauer, Institute for Geophysics and Meteorology, University of Cologne, D-50923 Cologne, Germany.

D. Reisenfeld, Department of Physics and Astronomy, University of Montana, Missoula, MT 59812, USA.

J. T. Steinberg, Los Alamos National Laboratory, Los Alamos, NM 87545 , USA

K. Szego, KFKI Research Institute for Particle and Nuclear Physics, KFKI-RMKI, P.O.Box 49, H-1525 Budapest, Hungary.

J. Vilppola, Department of Physical Sciences, University of Oulu, FIN-90014 Linnanmaa, Finland. 